\title{
RENDIMENTO DA PUPUNHEIRA EM FUNÇÃO DA DENSIDADE DE PLANTIO, DIÂMETRO DE CORTE E MANEJO DOS PERFILHOS NO VALE DO SÃO FRANCISCO
}

\author{
Effect of density, cut-off diameter classes and shoot number on \\ production and yield of irrigated plant peach palm at San Francisco River Valley
}

José Egidio Flori ${ }^{1}$, Geraldo Milanez de Resende ${ }^{1}$, Marcos Antônio Drumond ${ }^{1}$

\begin{abstract}
RESUMO
Com o objetivo de avaliar o rendimento de palmito de pupunheira irrigada em função da densidade de plantio, diâmetro de corte do estipe e o manejo dos perfilhos, instalou-se um experimento na Estação Experimental de Bebedouro da Embrapa Semi-Árido, Petrolina, PE. O delineamento utilizado foi de blocos ao acaso em parcelas subdivididas, sendo as parcelas constituídas por dois espaçamentos $\left(2,0 \times 1,0 \mathrm{~m}-\mathrm{E}_{1}\right.$ e 2,0 × 2,0 m- $\left.\mathrm{E}_{2}\right)$ e as subparcelas em arranjo fatorial $2 \times 2$, compreendendo dois manejos de perfilhos e duas classes de diâmetros de corte. As colheitas foram feitas a partir de 16 meses até os 69 meses após o plantio e realizadas com intervalos consecutivos de mais ou menos três meses. Pelos resultados, evidenciaram-se efeitos significativos para espaçamento, manejo de perfilhos e classe de diâmetro de corte. Não foram constatadas interações entre os tratamentos. Houve efeito significativo de espaçamento sobre o rendimento, diâmetro de corte, comprimento e peso médio do palmito. O rendimento e o peso do palmito da pupunheira foram de 545 e $434 \mathrm{~kg} \mathrm{ha}^{-1}$ ano $^{-1}$ e $147 \mathrm{~g}$ e $180 \mathrm{~g}$ nos espaçamentos $E_{1}$ e $E_{2}$, respectivamente. $O$ diâmetro de corte afetou a altura da planta e peso médio do palmito. O manejo de perfilho afetou o rendimento e o peso médio do palmito, com 532 e $446 \mathrm{~kg} \mathrm{ha}^{-1}$ e 177 e $149 \mathrm{~g}$ de palmito, respectivamente, no manejo de quatro e todos os perfilhos por planta. Pelos resultados, infere-se, como orientação técnica para os produtores de áreas irrigadas do Submédio São Francisco, o cultivo da pupunha no espaçamento de $2 \mathrm{~m} \mathrm{x} 1 \mathrm{~m}$, o manejo de quatro perfilhos por planta e o corte da planta com diâmetro variando de 10 a $12 \mathrm{~cm}$, a $30 \mathrm{~cm}$ do nível do solo.
\end{abstract}

Termos para indexação: Bactris gasipaes Kunth, palmito, semi-árido, produção.

\begin{abstract}
The objective of this experiment was to evaluate the effect of planting density, cut-off diameter classes and shoot number on heart of palm production on irrigated peach palm. The experimental layout was a randomized split-plot design with a factorial arrangement in the split-plot with four replicates. The whole plots were plant spacing $S_{1}-2 \times 1 \mathrm{~m}$ and $\mathrm{S}_{2}-2 \times 2 \mathrm{~m}$ and the subplots were $12 \mathrm{~m}^{2}$ (with factorial arrangement of two diameters and two shoot number). The harvesting was done from 16 to 69 months after planting with three months interval. The cut-off diameter class affected significantly the production. The yields of heart of palm at diameter classes $10 \mathrm{~cm}$ and $12 \mathrm{~cm}$ were 494 and $484 \mathrm{~kg} \mathrm{ha}^{-1}$ year $^{-1}$ and, respectively. The average weight of heart of palm for the cut-off diameters $10 \mathrm{~cm}$ and $12 \mathrm{~cm}$ were $149 \mathrm{~g}$ and $178 \mathrm{~g}$ and yield of heart of palm were 545 and $434 \mathrm{~kg} \mathrm{ha}^{-1} \mathrm{year}^{-1}$, respectively. Planting space affect all the parameters evaluated, except the average plant height. The results allow to point out, as information for the irrigated areas of the San Francisco Valley, the cultivation of peach palm spaced $2 \times 1 \mathrm{~m}$ and cut-off diameter of de stem from 10 to $12 \mathrm{~cm}$ about $30 \mathrm{~cm}$ up the grownd.
\end{abstract}

Index terms: Bactris gasipaes Kunth, heart-of-palm, semi-arid, yield.

(Recebido para publicação em 8 de abril de 2003 e aprovado em 7 de agosto de 2003)

\section{INTRODUÇÃO}

O Brasil é o maior produtor, exportador e consumidor de palmito do mundo. No ano de 1996, foram produzidos só de palmito extrativo $20.654 \mathrm{t}$, dos quais $4.853 \mathrm{t}$ foram exportados no valor de 25.766 milhões de dólares (IBGE, 1994). Para se ter uma idéia da dimensão dessa atividade, o faturamento médio anual do setor que é da ordem de 350 milhões de dólares, com geração de 8 mil empregos diretos e cerca de 25 mil indiretos (BOVI, 2000). Embora os números do setor sejam expressivos, observa-se que, no auge da produção, o Brasil chegou a produzir 202.000 t em 1989. Então se vê que o potencial dessa iguaria é muito grande. Atualmente dados não oficiais indicam uma estabilização na faixa de 20.000 t/ano - produção mantida graças ao 
palmito cultivado e ao manejo sustentável que vem sendo praticado nas reservas nativas (ASPECTOS..., 2003).

O palmito pode ser extraído de um grande número de gêneros e espécies de palmeiras. A abundância, a palatabilidade, a cor, o formato, a ausência de princípios tóxicos, o alto rendimento e a facilidade de extração são os principais fatores que fazem com que uma espécie seja preferida em relação à outra. Considerando esses atributos, palmeiras do gênero Euterpe (juçaras e açaizeiros) sempre foram as preferidas para a produção de palmito. A forma de exploração dessa e das demais palmeiras nativas continuam sendo uma atividade ainda extrativa e altamente predatória. No entanto, devido à alta taxa de exploração de palmeiras desse gênero e o relativamente baixo poder de regeneração das espécies de Euterpe, há atualmente falta de produto de boa qualidade. O resultado mais visível desse processo de exploração tem sido verificado na oferta de matéria-prima, que é cada vez menor, e na queda da qualidade do palmito.

$\mathrm{O}$ cultivo da pupunheira visando à produção de palmito vem se destacando como alternativa de produção sustentável e economicamente viável. Essa cultura apresenta algumas vantagens em relação a outras palmeiras produtoras de palmito como: precocidade de corte, boa produtividade, rusticidade, além de palmito de boa qualidade e sem escurecimento enzimático (VILLACHICA, 1996).

No Brasil, o cultivo da pupunheira foi intensificado a partir dos anos 90, sendo a estimativa da área plantada em 1996 de 5.600 ha (BOVI, 1997). Estima-se que se tenham atualmente cerca de 12 a 13 mil hectares plantados com essa palmeira, visando exclusivamente à produção de palmito, $50 \%$ dos quais já em fase de produção. O maior Estado produtor é São Paulo, com cerca de $25 \%$ do total implantado no País, seguido dos Estados: Espírito Santo, Rondônia, Pará, Bahia, Mato Grosso, Goiás, Minas Gerais, Rio Grande do Norte, Amazonas, Acre, Paraná, Santa Catarina e outros (BOVI, 2000). No Nordeste brasileiro, especificamente no Submédio do Vale do São Francisco, o cultivo da pupunheira foi iniciado em 1991 com plantas inermes (sem espinhos) provenientes da Embrapa Amazônia Ocidental, Manaus - AM. Os resultados da adaptação e produção realizados em Petrolina - PE confirmaram o potencial dessa cultura sob condições irrigadas (FLORI e D'OLIVEIRA, 1995, 1997).

Como a cultura ainda é nova no País, não existem orientações básicas para o seu cultivo, principal- mente para áreas irrigadas, como espaçamento, diâmetro de corte e manejo dos perfilhos (controle da brotação). Os estudos iniciais indicavam um espaçamento de $3 \mathrm{~m}$ x 1,5 m (MORA-URPI, 1989), porém verificou-se posteriormente que esse espaçamento era inadequado. Novos testes indicaram os espaçamentos de 1,5 m x 1,5 m e $2 \mathrm{~m}$ x $1 \mathrm{~m}$ como os ideais para a cultura (MORAURPI, 1989). Gomes (1987) avaliou a produção de palmito de pupunha no primeiro corte em dois espaçamentos de plantio (1,5 m x $1 \mathrm{~m}$ e $1 \mathrm{~m} \mathrm{x} 1 \mathrm{~m})$ e verificou que não houve diferença de produtividade entre espaçamentos. Clement (1995) verificou aumento de produtividade linearmente à medida que aumentava a densidade de plantio de 3.333 para 6.666 plantas/ha em diferentes progênies (variedades), nos primeiros 18 meses de colheita (35 meses após o plantio).

Em densidades entre 8.000 e 20.000 plantas/ha, a produção de palmito é máxima a partir da primeira colheita (JATIVA, 1996; VILLACHICA, 1996; BOGANTES et al., 1997). Entretanto, nos casos de plantio adensado, recomenda-se fazer o controle de perfilhos para que essa produtividade seja mantida ao longo do tempo, uma vez que o excesso de brotos tende a competir entre si, reduzindo a produtividade com o tempo. Por outro lado, em plantios com densidades iguais ou menores que 5.000 plantas/ha, a produção máxima é obtida a partir do segundo ou terceiro ano de colheita (MORA-URPI et al., 1997). Mora-Urpi et al. (1997) verificaram que após cinco anos de plantio ocorre uma confluência para o mesmo nível de produtividade em diferentes densidades.

Os estudos relativos ao diâmetro de corte do estipe ainda são incipientes. Clement et al. (1987) verificaram uma correlação entre o rendimento do palmito e o diâmetro da planta. Villachica (1996) recomenda o corte do estipe com $8 \mathrm{~cm}$ a $12 \mathrm{~cm}$ de diâmetro, e MoraUrpi et al. (1997) recomendam o corte acima de $9 \mathrm{~cm}$, medidos de 30 a $40 \mathrm{~cm}$ acima do colo da planta. Yuyama (1997) recomenda o corte do estipe quando aparecer o primeiro entrenó e quando a inserção da última folha verde no sentido de baixo para cima estiver acima dos $25 \mathrm{~cm}$ de altura do colo da planta.

O manejo dos perfilhos na touceira ainda tem sido uma prática recomendada com ressalvas pelos pesquisadores. Villachica (1996) recomenda o desbaste seletivo de perfilhos na touceira, ou seja, no momento da colheita, devem ser eliminados somente os perfilhos cujas bases de sustentação estejam na parte aérea do tronco da planta-mãe e aqueles que crescerão em direção à fileira adjacente. Mora-Urpi et al. (1997) recomendam 
o manejo de perfilhos na touceira com o objetivo de retardar a tendência natural da base da planta de se elevar em relação ao nível do solo. Outra observação feita por esses autores é que os perfilhos cujas bases de inserção no estipe for aéreo serão menos vigorosos, devido à falta de condições apropriadas para a regeneração dos seus sistemas radiculares.

Conduziu-se este trabalho com o objetivo de avaliar o efeito do espaçamento, da classe do diâmetro de corte e manejo de perfilhos na produtividade de palmito da pupunha irrigada no submédio São Francisco.

\section{MATERIAL E MÉTODOS}

O experimento foi conduzido na Estação Experimental da Embrapa Semi-Árido no Projeto de Bebedouro, Petrolina, PE. Foram utilizadas mudas sem espinhos com seis meses de idade com $30 \mathrm{~cm}$ de altura oriundas de um produtor comercial de mudas de Manaus, AM. O plantio foi realizado em março de 1995 em latossolo arenoso, com as seguintes características químicas na camada de $0-20 \mathrm{~cm}: \mathrm{K}=0,22$ $\mathrm{cmol}_{\mathrm{c}} / \mathrm{dm}^{3} ; \mathrm{Ca}=1,2 \mathrm{cmol}_{\mathrm{c}} / \mathrm{dm}^{3} ; \mathrm{Mg}=0,8 \mathrm{cmol}_{\mathrm{c}}$ $/ \mathrm{dm}^{3} ; \mathrm{Na}=0,02 \mathrm{cmol}_{\mathrm{c}} / \mathrm{dm}^{3} ; \mathrm{H}+\mathrm{Al}=0,65 \mathrm{cmol}_{\mathrm{c}}$ $/ \mathrm{dm}^{3} ; \mathrm{P}=20 \mathrm{mg} / \mathrm{kg} ; 1,43 \%$ de matéria orgânica; $81 \%$ de areia e 6.0 de $\mathrm{pH}$ em $\mathrm{H}_{2} 0$. O delineamento utilizado foi de blocos ao acaso com parcelas subdivididas com quatro repetições. A parcela principal constituiu-se dos espaçamentos $\left(\mathrm{E}_{1}=2 \mathrm{x} 1 \mathrm{~m}\right.$ 5.000 plantas $/$ ha e $E_{2}=2 \times 2 \mathrm{~m}-2.500$ plantas/ha). As subparcelas foram constituídas do manejo de perfilhos (planta com quatro e todos os perfilhos por touceira) combinados com duas classes de diâmetro de corte do estipe $(10$ e $12 \mathrm{~cm})$. A subparcela útil foi constituída por seis plantas $\left(12 \mathrm{~m}^{2}\right)$ e três plantas $\left(12 \mathrm{~m}^{2}\right)$ nos espaçamentos E1 (2 m x $\left.1 \mathrm{~m}\right)$ e E2 $(2 \mathrm{~m}$ x $2 \mathrm{~m})$, respectivamente, e a parcela útil, por 24 plantas $\left(48 \mathrm{~m}^{2}\right)$ e doze plantas $\left(48 \mathrm{~m}^{2}\right)$ nos espaçamentos $E_{1}$ $(2 \mathrm{~m} \times 1 \mathrm{~m})$ e $\mathrm{E}_{2}(2 \mathrm{~m} \times 2 \mathrm{~m})$, respectivamente. Cada subparcela útil tinha duas fileiras de plantas adjacentes com o mesmo espaçamento dessa, as quais tiveram a função de bordadura. A adubação de plantio foi de 100 e 300 gramas por metro linear na linha da cultura, respectivamente de $\mathrm{K}_{2} \mathrm{O}$ e $\mathrm{P}_{2} \mathrm{O}_{5}$. Após 30 dias do plantio, fez-se uma adubação de cobertura com $10 \mathrm{~g} / \mathrm{metro}$ linear de $\mathrm{N}$, que foi repetida a cada dois meses no primeiro ano. A adubação de produção foi realizada com a aplicação de 15 e $10 \mathrm{~g} / \mathrm{m}$ linear, respectivamente, para $\mathrm{N}$ e $\mathrm{K}_{2} \mathrm{O}$, a cada dois meses, e o fósforo com $10 \mathrm{~g} / \mathrm{m} \mathrm{li}$ near de $\mathrm{P}_{2} \mathrm{O}_{5}$, duas vezes ao ano. A irrigação foi condu- zida por sulcos de infiltração uma vez por semana, com a aplicação de uma lâmina média semanal de $35 \mathrm{~mm}$ d'água nos dois primeiros anos de cultivo; após esse período, a cultura foi irrigada por microaspersores de acordo com necessidade de água, usando-se como referência o método do tanque classe "A".

As características avaliadas foram diâmetro efetivo de corte (tomado a $30 \mathrm{~cm}$ do colo da planta), altura da planta (tomada do colo até a inserção da folha flecha - folha fechada), peso (g) e comprimento do palmito extra $(\mathrm{cm})$ (tolete tenro de $9 \mathrm{~cm}$ de comprimento, que mantinha a forma cilíndrica após retirada das bainhas mais fibrosas).

O manejo de perfilhos foi iniciado juntamente com a primeira colheita, aos 16 meses do plantio, e continuou sendo realizado sempre no momento do corte do estipe na touceira. O procedimento adotado no manejo de perfilhos foi eliminar os menores perfilhos que excediam o número pré-determinado de perfilhos. A colheita iniciou-se aos 16 meses após o plantio dez/96 e depois em fev/97 (23 meses), set/97 (30 meses), jan/98 (34 meses), mar/98 (36 meses), jun/98 (39 meses), set/98 (42 meses), dez/98 (45 meses), mar/99 (48 meses), nov/99 (56 meses), jun/00 (63 meses) e dez/00 (69 meses). Os dados foram analisados no programa Statistical Analysis System - SAS.

\section{RESULTADOS E DISCUSSÃO}

Pelos resultados, evidenciaram-se efeitos significativos para espaçamento, manejo de perfilhos e diâmetro de corte para as características avaliadas. Não foram constatadas interações entre os tratamentos (Tabela 1).

$\mathrm{O}$ espaçamento afetou todas as características mensuradas, exceto a altura da planta. $\mathrm{O}$ diâmetro de corte da planta afetou as características altura da planta e peso médio do palmito e não afetou o comprimento do palmito e o rendimento por hectare e por parcela. $\mathrm{O}$ manejo do perfilho afetou significativamente as características peso médio do palmito e a produtividade.

Os ganhos obtidos no peso médio do palmito com o espaçamento de 2 × 2 m não foram suficientes para compensar a produtividade do espaçamento de 2 $\mathrm{m} \times 1 \mathrm{~m}$. A produtividade acumulada do plantio no espaçamento de $2 \mathrm{~m}$ x $1 \mathrm{~m}$ apresentou-se maior no período de colheita avaliado.

No que se refere ao manejo de perfilhos, a diferença mais importante foi observada no rendimento, evidenciando a vantagem do controle de perfilhos na 
touceira como uma prática de manejo diferencial na cultura, visando ao ganho de produtividade (Tabela 1). Obteve-se no manejo da touceira com quatro perfilhos um aumento significativo no peso médio do palmito; esse ganho resultou em ganho significativo de rendimento. Evidentemente a recomendação dessa prática estará associada ao custo/benefício da mesma. De qualquer forma, quando essa prática é feita por operários treinados, torna-se simples e de baixo custo. $\mathrm{O}$ manejo dos perfilhos também poderá ser usado para fortalecer o sistema radicular da touceira, essa operação elemina os perfilhos que têm suas inserções no estipe acima do nível do solo, evitando-se, dessa forma, um afloramento precoce da touceira (VILLACHICA, 1996; MORA-URPI et al., 1997).

A produtividade média da pupunha deste trabalho, conduzida em solo arenoso, foi de cerca de $33 \%$ da produtividade do solo argiloso (1.86 t/ha/ano), trabalho esse conduzido por Flori et al. (2001). Uma causa bastante provável para um menor rendimento da cultura no solo arenoso foi a presença de lençol freático bastante superficial (média de 0,80 m), acarretando um nível mais alto de condutividade elétrica do extrato de saturação desse solo. Outras características inerentes desse solo que também afetaram seu potencial produtivo foram a baixa CTC, baixa retenção de água e baixo teor de matéria orgânica.

TABELA 1 - Altura e diâmetro do estipe da planta e peso médio, comprimento e rendimento do palmito extra da pupunheira (1996 - 2000). Embrapa Semi-Àrido, Petrolina, PE, 2000.

\begin{tabular}{|c|c|c|c|c|c|c|}
\hline \multirow{2}{*}{ Tratamentos } & \multicolumn{2}{|c|}{ Estipe (planta) } & \multicolumn{4}{|c|}{ Palmito Extra** } \\
\hline & Altura (m) & $\begin{array}{l}\text { Diâmetro* } \\
\quad(\mathbf{c m})\end{array}$ & $\begin{array}{c}\text { Peso médio } \\
\text { (g) }\end{array}$ & $\begin{array}{c}\text { Comprimento } \\
\text { (cm) }\end{array}$ & $\begin{array}{c}\text { Rendimento } \\
\text { (kg/ha/ano) }\end{array}$ & $\begin{array}{c}\text { Rendimento } \\
\text { parcela }(\mathrm{g})\end{array}$ \\
\hline \multicolumn{7}{|c|}{ Diâmetros de corte } \\
\hline $10 \mathrm{~cm}$ & $2,37 \mathrm{~B}$ & $10,4 * \mathrm{~B}$ & $149 \mathrm{~B}$ & $32 \mathrm{~A}$ & $494 \mathrm{~A}$ & $594 \mathrm{~A}$ \\
\hline $12 \mathrm{~cm}$ & $2,80 \mathrm{~A}$ & $11,9 * \mathrm{~A}$ & $178 \mathrm{~A}$ & $33 \mathrm{~A}$ & $484 \mathrm{~A}$ & $580 \mathrm{~A}$ \\
\hline \multicolumn{7}{|l|}{ Espaçamentos } \\
\hline $\mathrm{E}_{1} 2 \times 1 \mathrm{~m}$ & $2,49 \mathrm{~A}$ & $10,76 \mathrm{~B}$ & $147 \mathrm{~B}$ & $31 \mathrm{~B}$ & $545 \mathrm{~A}$ & $654 \mathrm{~A}$ \\
\hline $\mathrm{E}_{2} 2 \times 2 \mathrm{~m}$ & $2,68 \mathrm{~A}$ & $11,59 \mathrm{~A}$ & $180 \mathrm{~A}$ & $34 \mathrm{~A}$ & $434 \mathrm{~B}$ & $520 \mathrm{~B}$ \\
\hline \multicolumn{7}{|c|}{ Manejos dos perfilhos/planta } \\
\hline 4 perfilhos & $2,52 \mathrm{~A}$ & $11,31 \mathrm{~A}$ & $177 \mathrm{~A}$ & $33 \mathrm{~A}$ & $532 \mathrm{~A}$ & $638 \mathrm{~A}$ \\
\hline Todos perfilhos & $2,65 \mathrm{~A}$ & $11,03 \mathrm{~A}$ & $149 \mathrm{~B}$ & $32 \mathrm{~A}$ & $446 \mathrm{~B}$ & $535 \mathrm{~B}$ \\
\hline C.V.(\%) & 10,61 & 13,81 & 14,96 & 10,93 & 23,09 & 23,09 \\
\hline
\end{tabular}

Médias nas colunas por tratamento seguidas da mesma letra não diferem entre si pelo teste Tukey $(\mathbf{p}<0,05)$.

*Média do diâmetro efetivo de corte;

**Palmito macio e cilíndrico com 9 cm de comprimento. 


\section{CONCLUSÕES}

Pelos resultados obtidos, infere-se, como orientação técnica para os produtores das áreas irrigadas do Submédio São Francisco, o cultivo da pupunheira no espaçamento de $2 \mathrm{~m} \times 1 \mathrm{~m}$, com manejo de quatro perfilhos por planta e o corte da planta com diâmetro variando de 10 a $12 \mathrm{~cm}$.

\section{REFERÊNCIAS BIBLIOGRÁFICAS}

ASPECTOS econômicos. Disponível em: <http:/ /www.unicamp.br/nipe/rbma/pal_eco.htm>. Acesso em: 22 set. 2003.

BOGANTES, A.; MORA-URPI, J.; ARROYO, C. Densidades de siembra. In: CURSO INTERNATIONAL SOBRE O CULTIVO DE PEJIBAYE PARA PRODUCCION DE PALMITO, 2., 1997, San José. Anais... San José: Universidade de Costa Rica, 1997. p. 12-14.

BOVI, M. A. Expansão do cultivo da pupunheira para palmito no Brasil. In: CONGRESSO BRASILEIRO DE OLERICULTURA, 38., 1997, Manaus. Suplemento... Brasília: SOB, 1997. p. 183-185.

BOVI, M. A. O agronegócio palmito de pupunha. O Agronômico, Campinas, v. 52, n. 1, 2000. Disponível em: <http://www.iac.sp.gov.br/new/oagronomico/521 informaçoestecnicas/pupunha/pdf $>$. Acesso em: 23 set. 2003.

CLEMENT, C. R. Growth and genetic analysis of pejibaye (Bactris gasipaes Kunth, Palmae) in Hawai. 1995. 138 f. Thesis (Ph.D.) - University of Hawai, Honolulu, 1995.

CLEMENT, C. R.; CHAVES FLORES, W. B.; GOMES, J. B. M. Considerações sobre a pupunha (Bactris gasipaes K.). In: ENCONTRO NACIONAL DE PESQUISADORES DE PALMITO, 1., 1987, Curitiba. Anais... Curitiba: EMBRAPA-CNPF, 1987. p. 226-266. (Documentos, 19).

FLORI, J. E.; D'OLIVEIRA, L. O. B. O cultivo da pupunha sob irrigação no semi-árido do Nordeste brasileiro. Petrolina, PE: EMBRAPA-CPATSA, 1995. (Comunicado técnico, 63).
FLORI, J. E.; D'OLIVEIRA, L. O. B. O cultivo da pupunha irrigada no semi-árido. Petrolina, PE: EMBRAPA-CPATSA, 1997. (Instruções técnicas, 2).

FLORI, J. E.; RESENDE, G. M. de R.; DRUMOND, M. A. Rendimento do palmito de pupunha em função da densidade de plantio, diâmetro de corte e manejo de perfilhos, no Vale do São Francisco. Horticultura Brasileira, Brasília, v. 19, n. 2, p. 140-143, 2001.

GOMES, J. B. M.; MENEZES, J. M. T.; VIANA FILHO, P. Efeito de níveis e adubação e espaçamento na produção de palmito. In: ENCONTRO NACIONAL DE PESQUISADORES DE PALMITO, 1., 1987, Curitiba. Anais... Curitiba: EMBRAPA-CNPF, 1987. p. 261-269.

INSTITUTO BRASILEIRO DE GEOGRAFIA E ESTATÍSTICA. Anuário estatístico do Brasil. Rio de Janeiro, 1994. v. 52, 920 p.

JATIVA, M. Investigaciones en chontaduro (Bactris gasipaes Kunth) Ecuador. In: WORKSHOP SOBRE AS CULTURAS DE CUPUAÇU E PUPUNHA NA AMOZÔNIA, 1., 1996, Manaus. Anais... Manaus: EMBRAPA-CPAA, 1996. p. 50-58.

MORA-URPI, J. Densidade de siembra para producción de palmito. Boletim Pejibaye, San José, v. 1, n. 1, p. 10-12, ene/mar. 1989.

MORA-URPI, J.; WEBER, J. C.; CLEMENT, C. R. Peach Palm (Bactris gasipaes Kunth): promoting the conservation and use of underutilized and neglected crops. Gastersleben; Rome: Institute of Plant Genetics and Crop Plant Research; International Plant Genetic Resources Institute, 1997. 83 p.

VILLACHICA, H. Cultivo del pijuayo (Bactris gasipaes Kunth) para palmito en la Amazônia. Lima: TCA, 1996. $153 \mathrm{p}$.

YUYAMA, K. Sistemas de cultivo para produção de palmito da pupunheira. In: CONGRESSO BRASILEIRO DE OLERICULTURA, 38., 1997, Manaus. Suplemento... Brasília: SOB, 1997. p. 191199. 